\title{
Laser-Assisted Endoscopic Cricotracheal Stenosis Resection (CTSR) in Paediatric Congenital Cartilaginous Subglottic Stenosis
}

\author{
Jaber Alshammari, Abdullah Arafat $\mathbb{D}$, and Mohammed Halawani \\ Division of Otolaryngology Head \& Neck Surgery, King Abdullah Specialized Children Hospital (KASCH), \\ King Abdulaziz Medical City (KAMC), National Guard Health Affairs (NGHA), Riyadh, Saudi Arabia
}

Correspondence should be addressed to Abdullah Arafat; arafat.as@outlook.sa

Received 1 May 2020; Accepted 8 June 2020; Published 29 June 2020

Academic Editor: Gerd J. Ridder

Copyright (C) 2020 Jaber Alshammari et al. This is an open access article distributed under the Creative Commons Attribution License, which permits unrestricted use, distribution, and reproduction in any medium, provided the original work is properly cited.

\begin{abstract}
Subglottic stenosis (SGS) in children can be a congenital condition or acquired through injury such as from prolonged intubation. Surgeons face challenges in choosing the best SGS treatment for a particular patient because of variability in the success rate of each technique. Conventional open surgical resection and reconstruction have been proven effective but, in recent years, endoscopic surgery has become more prevalent as it eliminates the incision and reduces the surgery time and subsequent hospital stay. The purpose of this retrospective case study was to report on an endoscopic technique using a $\mathrm{CO}_{2}$ laser for cricotracheal stenosis resection (CTSR) for high-grade congenital SGS. From forty-five paediatric patients who underwent endoscopic intervention as a primary modality of treatment for high-grade SGS in a tertiary referral centre, a total of eight patients who met the inclusion criteria have been included in our study. This small patient series is the first to use a $\mathrm{CO}_{2}$ laser alone as a single excision tool to eliminate complex congenital SGS and restore airway patency. The procedure's goal was to return the airway to an early stage of postintubation injury prior to scar formation; therefore, surgical sessions at intervals of 2-3 weeks were performed to ensure natural epithelization, to remove any granulation tissue, and manage fibrosis. Successful treatment was defined as a resolution of symptoms, restoration of a normal patent airway with no stenosis, and decannulation. The success rate was $75 \%$. Two outcomes need to be highlighted. First, the $\mathrm{CO}_{2}$ laser should be reconsidered as an excision tool for congenital SGS because of its low risk of exacerbating preexisting stenosis. It allows the surgeon to restore and augment the airway without the need for open surgery or dilatation. Second, the shorter interval between procedures is crucial for controlling the healing process and making sure that it is proceeding properly.
\end{abstract}

\section{Introduction}

The subglottic region is highly vulnerable to instrumentation and pathological processes. Children are either born with laryngotracheal stenosis or acquire it during their development. The subglottis plays a crucial role in children because it serves as the main site of airway narrowing [1]. Cricoid cartilage malformation is one of the major characteristics of stenosis in congenital cases. Prolonged intubation is a risk factor for noncongenital subglottic stenosis (SGS) and accounts for $90 \%$ of cases [2]. SGS treatment presents a significant challenge in children. Cricotracheal resection (CTR) or open reconstruction with laryngotracheal reconstruction (LTR) has been proven an effective and safe SGS treatment option for the paediatric population. However, the use of endoscopic techniques in the management of SGS treatment has grown over the last decades. Additionally, treatment of SGS in the paediatric population with less-invasive surgical procedures has advanced over time. Several endoscopic procedures such as stent placement, microdissection, endoscopic laser resection, and endoscopic dilation are applied either as a primary treatment strategy, or to compliment an open reconstructive procedure. These procedures offer three potential benefits: decrease in operation time, decrease in the admission length, and prevention of external incisions. The rate of success of the endoscopic approach is also increased by use of adjuvant treatments such as administration of mitomycin-C and 
steroids [3]. Surgeons face challenges in making decisions about the most efficient type of modality or the combination of modalities for SGS treatment due to variation in the success rates of each technique. Though no strict criteria for managing SGS have been codified, the endoscopic approach has been limited to low-grade (grades I and II), noncircumferential, and short-segment SGS [4-9]. In spite of all these recommendations and case selection, endoscopic management still shows recurrent scar formation rates of $40 \%$ to $70 \%$ [10]. In grade III SGS, the success rate declined to $13 \%$, indicating that endoscopic treatment can seldom restore a subglottis to an anatomically normal state.

Studies have demonstrated only a minor role for laser treatment in pure congenital SGS except for a few trials of cricoid split and when using balloon dilatation [11]. The objective of this study was to describe an endoscopic technique using a $\mathrm{CO} 2$ laser for cricotracheal stenosis resection (CTSR) to manage high-grade complex SGS and to assess this technique in high-grade congenital SGS without open procedure. To the best of our knowledge, this is the first study to discuss the use of an endoscopic CO2 laser as a single excision tool to eliminate high-grade congenital SGS and restore airway integrity.

\section{Materials and Methods}

Ethical approval of the study was provided by the King Abdullah International Medical Research Centre (KAIMRC) with research protocol RC16/208/R.

2.1. Patients. In this retrospective case series, we reviewed the charts of 45 paediatric patients (ranging in age from one month to 14 years) who underwent endoscopic intervention as a primary modality of treatment for high-grade SGS in a tertiary referral centre (King Abdulaziz Medical City, Riyadh, Saudi Arabia) from January 1, 2011 to October 31, 2019. All children who had a workable diagnosis of congenital SGS and who underwent at least one endoscopic intervention as the primary treatment modality without previous primary open laryngotracheoplasty were included in the study. After applying our inclusion criteria, eight patients with congenital SGS out of 45 SGS cases managed by $\mathrm{CO}_{2}$ laser-assisted endoscopic CTSR at our institution were included in this study. The patients had been referred from different secondary hospitals in Saudi Arabia and were tracheostomized with a diagnosis of SGS based on direct laryngobronchoscopy (DLB) findings. Definitive or successful treatment was defined as a resolution of symptoms, restoration of a normal patent airway with no stenosis, and decannulation.

Children who were diagnosed with acquired SGS were excluded. Charts were reviewed for the number of primary surgeons, age, gender, history of prematurity, significant medical comorbidities, descriptions of SGS, types and timing of interventions for SGS, and airway status at the last available follow-up visit. The diagnosis and description of SGS were performed by the paediatric otolaryngologists in the operating room at the time of the direct laryngobronchoscopy (DLB). Information on the characteristics of the SGS was taken from the surgeon's description in the surgical notes. Three paediatric otolaryngologists, one consultant and two fellows, participated in the surgeries, and the consultant was always there to confirm the description. The Myer-Cotton grading system was used to standardize descriptions of the degree of the SGS. The follow-up lasted for at least three years after the last intervention.

2.2. Statistical Analysis. All collected data were analyzed with SPSS version 17 using the mean \pm SD for the quantitative variables and frequencies and percent for the qualitative variables. All statistical analyses of the qualitative data before and after endoscopic treatment of SGS were performed using McNemar's test. Differences were considered to be significant when $p$ was $<0.05$ and highly significant when $p$ was $<0.01$.

2.3. Surgical Technique. Under a spontaneous ventilation general anaesthesia technique, the patient's larynx was exposed using a rigid laryngoscope with lateral ports for ventilation. This technique permitted the surgical field to be free of ventilation material during the procedure. Endoscopy was undertaken to examine the area of stenosis and additional synchronous lesions. Photographic documentation of the stenotic segment was obtained using a 2.7 or $4 \mathrm{~mm}$ and 0 degree Karl Storz telescope. Descriptions of the grade, site, craniocaudal extension, distance from the vocal folds, and texture of the stenosis were crucial. The Cotton-Myer scale was used to estimate the grade of stenosis. A vocal cord spreader was then applied to expose the SGS (Figure 1). An UltraPulse ${ }^{\circledR}$ DUO CO2 surgical laser (Lumenis $\mathrm{GmbH}$, Dreieichenhain, Germany) with a microspot, ultrapulse mode and a power of $125 \mathrm{mj} / \mathrm{cm}^{2}$ was used to excise the stenosis by peeling off the cricoid cartilage in a craniocaudal direction (Figure 2). The perichondrium of the cricoid cartilage is difficult to completely preserve but the aim was to avoid complete cartilage denudation (Figure 2). Mechanical dilatation is not part of this procedure. Haemostasis was achieved with pledgets soaked in adrenalin to keep the field bloodless. Submucosal injections of a long-acting corticosteroid, commonly triamcinolone acetonide $(40 \mathrm{mg} / \mathrm{ml})$, were done above and below the level of the excised stenosis. The laryngeal stent was then inserted and fixed by Lichtenberger needle to prevent restenosis by granulation tissue (Figure 3). The area was cooled and cleaned of any debris with saline-wetted pledgets. Patients were monitored in the paediatric intensive care unit for 24 to 48 hours using a noninvasive ventilation system. All patients received acetaminophen and nonsteroidal anti-inflammatory drugs to control pain. Systemic steroids (prednisolone, $1-2 \mathrm{mg} / \mathrm{kg} /$ day) were administered for ten days. Proton pump inhibitors (esomeprazole, $2 \mathrm{mg} / \mathrm{kg} /$ day) were prescribed for 28 days. Patients were fed postoperatively through a nasogastric tube and then gradually shifted to oral intake. The aim of this procedure was to return the airway to an early stage of postintubation injury prior to scar formation. Then, subsequent surgical sessions at intervals of 2-3 weeks were performed to remove the stent and to ensure that natural 


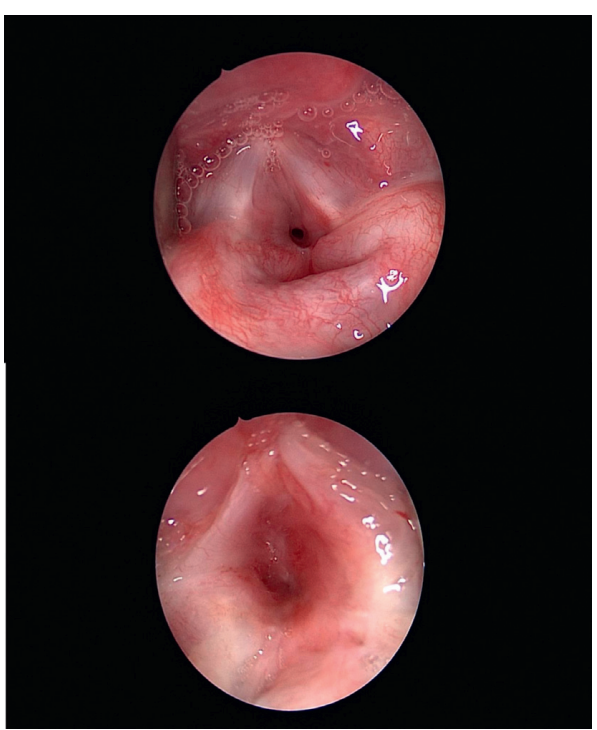

FIgure 1: Congenital subglottic stenosis.

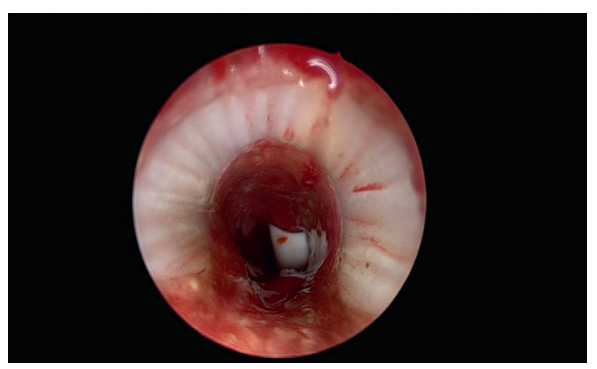

FIgURE 2: Excision of SGS by laser.

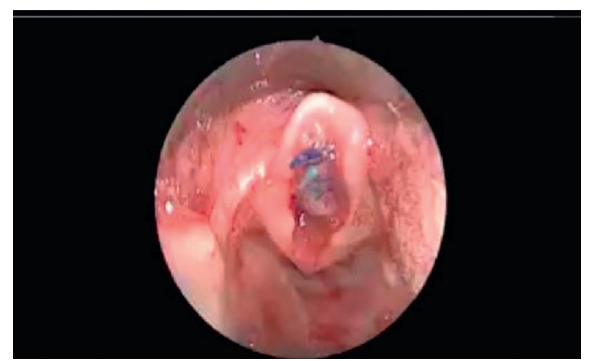

Figure 3: Insertion of endolaryngeal stent.

epithelization was proceeding favourably, to remove any granulation tissue and to manage early recurrent fibrosis. Mucosal healing from both edges was measured and documented regularly during all sessions until complete epithelization of the area occurred (Figure 2). Allowing scar tissue to accumulate and mature will negate the outcome and require the surgeon to start over from the beginning. After complete epithelization, the follow-up diagnostic laryngoscopy continued every six months for 48 months.

\section{Results}

After applying our inclusion criteria, only eight patients out of 45 SGS cases managed by $\mathrm{CO}_{2}$ laser-assisted endoscopic CTSR at our institution were included in this study. Four male and four female patients with an average age of 11.5 months (range, 0.1-24 months) were identified. Five patients had a history of concurrent pulmonary disease and three had a history of gastroesophageal reflux. Three patients reported neurological disease and one had congenital heart disease (Table 1). Presenting symptoms of SGS included severe dyspnoea, biphasic continuous stridor, dysphonia, and cough. Two patients (16\%) were categorized as having idiopathic stenosis. When a quantitative airway diameter was reported, the Cotton-Myer grading scale was applied to estimate subglottic diameter. All eight patients (100\%) had grade III stenosis (defined as $71 \%-99 \%$ stenosis). The craniocaudal extensions ranged from $8 \mathrm{~mm}$ to $15 \mathrm{~mm}$ with an average length of $11.6 \mathrm{~mm}$ (Table 2). All patients had circumferential stenosis and all of them had a tracheostomy surgery. The eight patients underwent 21 surgical procedures. Three of eight patients (37.5\%) required a single procedure with a mean surgical duration of 1.4 hours. Five patients $(62.5 \%)$ required multiple surgeries (3-4 procedures). Of the patients who required further procedures, the mean interval between surgeries was 3.1 weeks. The mean hospital stay was 4 days (range, 2-10 days) for each procedure.

Definitive or successful treatment was defined as a resolution of symptoms, restoration of a normal patent airway with no stenosis, and decannulation. Patients who have been treated successfully were decannulated within 4 to 5 weeks after the last surgical session with a mean of 31 days. The success rate was $75 \%$. Two patients (25\%) had residual grades I-II SGS and underwent open laryngotracheal reconstruction procedure with anterior and posterior grafts. Using the Friedman test to compare preoperative and stenotic grade showed statistical significance with a $p$ value of 0.0082 (Table 2). All the patients who were decannulated were able to tolerate regular oral feeding. The median follow-up duration for all patients from the date of the first procedure to the last clinical visit was three years (range, 2-4.5 years).

\section{Discussion}

The subglottis, as a junction between two embryological growth centres, is particularly vulnerable to instrumentation and pathological processes [4]. It can be affected by a tenuous blood supply or microcirculation [12]. Other factors previously postulated to potentiate stenosis include exposure to gastric reflux, turbulent airflow, and presence of a complete cartilage ring [12]. SGS, regardless of its aetiology, is a difficult problem to treat. Treatment has traditionally involved open procedures such as LTR or PCTR [1, 2]. Although open procedures have high rates of decannulation [6], they also have the potential for severe complications $[3,13]$. While endoscopic procedures avoid these complications, extreme variability in results is reported in the literature [10]. The recurrence of scar formation has shown high rates of $40 \%$ to $70 \%$ in endoscopic management [4]. To obtain good results, therefore, conservative endoscopic modalities have been advocated [4-8]. In 1982, Simpson et al. concluded that factors associated with poor endoscopic results or failure included circumferential scarring with cicatricial contracture and scarring wider than one $\mathrm{cm}$ in the 
TABle 1: Demographics, length of stenosis, preop and postop grade of SGS, medical Hx, and number of procedures (patients undergoing open surgery marked in red).

\begin{tabular}{|c|c|c|c|c|c|c|c|c|}
\hline & $\begin{array}{c}\text { Age at } \\
\text { diagnosis }\end{array}$ & $\begin{array}{l}\text { Current } \\
\text { age }\end{array}$ & Gender & $\begin{array}{l}\text { Length of stenosis } \\
(\mathrm{mm})\end{array}$ & $\begin{array}{l}\text { SGS } \\
\text { preop }\end{array}$ & $\begin{array}{c}\text { SGS } \\
\text { postop }\end{array}$ & Medical HX & $\begin{array}{l}\text { Number of } \\
\text { procedures }\end{array}$ \\
\hline $\mathrm{Pt} \# 1$ & 11 months & 5.4 years & $\mathrm{F}$ & 9 & 3 & 0 & Stickler syndrome & 1 \\
\hline $\mathrm{Pt} \# 2$ & 12 months & 4.2 years & $\mathrm{M}$ & 8 & 3 & 2 & $\begin{array}{c}\text { Neurological } \\
\text { hypotonia }\end{array}$ & 4 \\
\hline $\mathrm{Pt} \# 3$ & 15 months & 3.3 years & $\mathrm{F}$ & 12 & 3 & 1 & None & 1 \\
\hline $\mathrm{Pt} \# 4$ & 5 months & 2.8 years & M & 13 & 3 & 1 & Cervical teratoma & 3 \\
\hline $\mathrm{Pt} \# 5$ & 24 months & 4 years & M & 10 & 3 & 1 & Neurological disease & 1 \\
\hline $\mathrm{Pt} \# 6$ & 16 months & 5 years & $\mathrm{F}$ & 12 & 3 & 0 & Neurological disease & 3 \\
\hline $\mathrm{Pt} \# 7$ & 9 months & 4 years & M & 15 & 3 & 2 & Cardiac disease & 4 \\
\hline $\mathrm{Pt} \# 8$ & 1 month & 4 years & $\mathrm{F}$ & 14 & 3 & 0 & None & 4 \\
\hline
\end{tabular}

TABle 2: Stridor and endoscopic demographic grading of SGS before and after CTSR.

\begin{tabular}{lccc}
\hline Variable & Before CTSR & After CTSR & $p$ value \\
\hline Stridor & & & \\
No & $0(0.0 \%)$ & $6(75 \%)$ & \\
Yes & $8(100.0 \%)$ & $2(25 \%)$ & $<0.01$ \\
\hline Grade of stenosis & & $6(75 \%)$ & \\
Grade 0 & 0 & 0 & \\
Grade I & 0 & $2(25 \%)$ & \\
Grade II & 0 & 0 & $<0.01$ \\
Grade III & $8(100 \%)$ & 0 & \\
Grade IV & 0 & &
\end{tabular}

vertical dimension [11]. Triglia et al. advised laser resection only for grade I SGS less than $5 \mathrm{~mm}$ in length [8]. Monnier et al. reviewed 100 patients treated solely by endoscopic means for laryngotracheal stenosis $[14,15]$. The postoperative results showed that the improvement declined from $92 \%$ for grade I to $46 \%$ for grade II and $13 \%$ for grade III stenosis. When compared with open surgery for more severe grades of stenosis, the results of endoscopy were much less favourable [6]. It has been shown that $\mathrm{CO}_{2}$ lasers with pulsed technology vaporize tissue quickly, avoid thermal damage, permit constant tissue ablation, and avoid charring and scarring [14-16]. It was thought that laser energy would heat the surrounding tissues and might cause additional scar formation [9], but Hseu et al. reported in 2014 that $\mathrm{CO}_{2}$ laser surgery was not associated with worse outcomes or more frequent dilations [4]. This unprecedented finding encouraged the use of $\mathrm{CO}_{2}$ lasers not only for the radial incision but also as a "knife" to excise the tissue causing SGS. In addition, the suspension system of the $\mathrm{CO}_{2}$ laser (micromanipulator) is very sensitive, thereby allowing precise manipulation of the laser beam to resect the fibrosis en bloc. We achieved endoscopic excision of congenital SGS solely with a $\mathrm{CO}_{2}$ laser and ultimately augmented the airway without any mechanical dilatations. We did encounter one complication of minimal airway fire managed intraoperatively; also, the vocal cord shape in our series seemed more thickened and was covered by respiratory epithelium instead of stratified squamous epithelium. The healing process was monitored closely by performing therapeutic microlaryngoscopy every 2-3 weeks (Figure 4). Our findings concur with those of Hseu et al. that laser surgery was not associated with worse

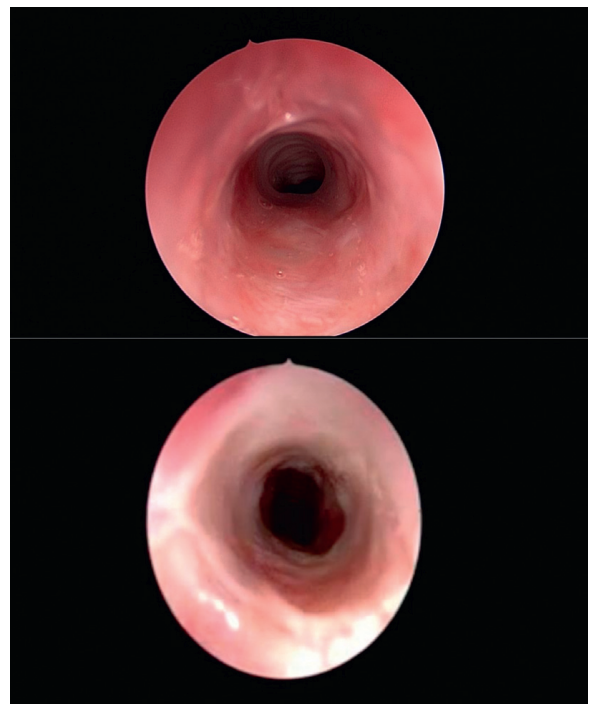

FIgURE 4: Endoscopic photographs showing the improvement in the extension of stenosis and the degree of severity in SGS that occurred in one patient during each surgical session of CTSR.

outcomes; on the contrary, there was obvious improvement [4]. We called this technique CTSR. In CTSR, the fibrous stenotic portion was resected as in open PCTR, but without affecting the integrity of the anatomical framework, and the lumen of the airway was augmented and restored as in open LTR with a minimally invasive endoscopic technique.

The present study is a preliminary report to assess the technique for correcting high-grade circumferential SGS. Despite the selection criteria, we achieved a success rate of $75 \%$ and only two patients (25\%) continued to present residual grade II SGS. Reports in the literature following primary endoscopic treatment of LTS showed success rates from $40 \%$ to $94 \%$, depending on the appropriateness of the indication [4-13]. The number of subjects in this report was limited; therefore, some comparisons will be underpowered. The average number of surgical sessions required for each patient was three. $38 \%$ of patients required only a single procedure and $62 \%$ required multiple surgeries (3-4 procedures), which are comparable to the results of other studies that were more conservative in using $\mathrm{CO}_{2}$ lasers [4]. One of the drawbacks of our approach is the need for multiple surgical sessions with its consequences of 
psychological trauma to paediatric patients although this can be balanced with the shorter hospital stay and less-invasive care compared to other open techniques. The major difference in this technique is the interval between surgical sessions. The anticipated interval between procedures is set at 2-3 weeks to control the natural healing process, remove granulation tissue, and manage recurrent stenosis. In other algorithms, it sometimes takes months before the patient presents in need of urgent intervention [4]. In our opinion, this interval is too long. It can negate the beneficial outcomes of the surgery and cause the trachea to revert to its previous degree of stenosis or worse. In spite of the comorbidities in some patients, which might be contraindications to open surgery, this technique was performed successfully. Of course, the number of subjects was small, owing to the rarity of the disease and the selection criteria, but it is considered as a preliminary report to assess this technique in acquired high-grade, circumferential SGS endoscopically. This small patient series is the first to use a $\mathrm{CO}_{2}$ laser alone as a single excision tool to eliminate complex SGS and restore airway patency. Finally, two outcomes need to be highlighted. First, the $\mathrm{CO}_{2}$ laser should be reconsidered as an excision tool for SGS without much risk of worsening preexisting stenosis. It provides a chance to restore and augment the airway without the need for open surgery or dilatation. Second, the shorter interval between procedures is crucial for controlling the healing process and making sure that it is proceeding properly.

\section{Conclusions}

SGS is a difficult problem for the otolaryngologist to address and is complicated by the fact that there is no standard algorithm for treatment. This study was designed to assess a new endoscopic technique using a $\mathrm{CO}_{2}$ laser to resect cricotracheal stenosis without affecting the airway framework integrity. In this study, it was used to treat high-grade, circumferential SGS. The success rate of this small sample is promising. This is the first patient series reported to date using a $\mathrm{CO}_{2}$ laser as the sole excision tool to eliminate SGS without any mechanical dilatation. This innovative surgical approach might change the consensus for the endoscopic surgical management of SGS. Further larger studies are needed to confirm its efficacy and safety.

\section{Data Availability}

The data used to support the findings of this study are available from the corresponding author upon request.

\section{Ethical Approval}

This paper was reviewed and ethically approved by King Abdullah International Medical Research Center, Riyadh, Saudi Arabia.

\section{Conflicts of Interest}

The authors declare that they have no conflicts of interest.

\section{Acknowledgments}

The authors would like to thank all the residents and nurses who participated in the study. Special thanks are due to the Otorhinolaryngology Department at King Abdulaziz Medical City and the Medical Education Department at King Saud bin Abdulaziz University for Health Sciences for their technical support.

\section{References}

[1] R. T. Cotton, "Management of subglottic stenosis," Otolaryngologic Clinics of North America, vol. 33, no. 1, pp. 111-130, 2000.

[2] K. Sandu and P. Monnier, "Cricotracheal resection," Otolaryngologic Clinics of North America, vol. 41, no. 5, pp. 981-998, 2008.

[3] N. Agrawal, M. Black, and G. Morrison, "Ten-year review of laryngotracheal reconstruction for paediatric airway stenosis," International Journal of Pediatric Otorhinolaryngology, vol. 71, no. 5, pp. 699-703, 2007.

[4] A. F. Hseu, M. S. Benninger, T. M. Haffey, and R. Lorenz, "Subglottic stenosis: a ten-year review of treatment outcomes," The Laryngoscope, vol. 124, no. 3, pp. 736-741, 2014.

[5] C. B. Simpson and J. C. James, "The efficacy of mitomycin-C in the treatment of laryngotracheal stenosis," The Laryngoscope, vol. 116, no. 10, pp. 1923-1925, 2006.

[6] P. Monnier, M. George, M.-L. Monod, and F. Lang, "The role of the $\mathrm{CO} 2$ laser in the management of laryngotracheal stenosis: a survey of 100 cases," European Archives of Oto-RhinoLaryngology, vol. 262, no. 8, pp. 602-608, 2005.

[7] M. J. Rutter, A. P. Cohen, and A. de Alarcon, "Endoscopic airway management in children," Current Opinion in Otolaryngology \& Head and Neck Surgery, vol. 16, no. 6, pp. 525-529, 2008.

[8] J. M. Triglia, R. Nicollas, and S. Roman, "Management of subglottic stenosis in infancy and childhood," European Archives of Oto-Rhino-Laryngology, vol. 257, pp. 328-335, 2000.

[9] T. D. Otteson, V. C. Sandulache, M. Barsic, G. M. DiSilvio, P. A. Hebda, and J. E. Dohar, "Acute and chronic changes in the subglottis induced by graded carbon dioxide laser injury in the rabbit airway," Archives of Otolaryngology-Head \& Neck Surgery, vol. 134, no. 7, pp. 694-702, 2008.

[10] N. Hirshoren and R. Eliashar, "Wound-healing modulation in upper airway stenosis-myths and facts," Head \& Neck, vol. 31, no. 1, pp. 111-126, 2009.

[11] D. J. Rogers and C. J. Hartnick, "Endoscopic CO2 laser laryngofissure in pediatric laryngotracheal reconstruction," International Journal of Pediatric Otorhinolaryngology, vol. 77, no. 5, pp. 850-853, 2013.

[12] A. M. Quesnel, G. S. Lee, R. C. Nuss, M. S. Volk, D. T. Jones, and R. Rahbar, "Minimally invasive endoscopic management of subglottic stenosis in children: success and failure," International Journal of Pediatric Otorhinolaryngology, vol. 75, no. 5, pp. 652-656, 2011.

[13] G. T. Simpson, M. S. Strong, G. B. Healy, S. M. Shapshay, and C. W. Vaughan, "Predictive factors of success or failure in the endoscopic management of laryngeal and tracheal stenosis," Annals of Otology, Rhinology \& Laryngology, vol. 91, no. 4, pp. 384-388, 1982.

[14] P. Monnier, "Equipment and instrumentation for diagnostic and therapeutic endoscopy," in Paediatric Airway Surgery Management of Laryngotracheal Stenosis in Infants and 
Children, P. Monnier, Ed., pp. 45-75, Springer, Berlin, Germany, 2011.

[15] J. Alshammari, A. A. Alkhunaizi, and A. S. Arafat, "Tertiary center experience with primary endoscopic laryngoplasty in pediatric acquired subglottic stenosis and literature review," International Journal of Pediatrics and Adolescent Medicine, vol. 4, no. 1, pp. 33-37, 2017.

[16] H. Moseley and V. Oswal, "Laser biophysics," in Principles and Practice of Lasers in Otorhinolaryngology and Head and Neck Surgery, V. Oswal and M. Remacle, Eds., pp. 5-30, Kugler, The Hague, Netherlands, 2002. 